\title{
Activation of the Arabidopsis thaliana Mitogen-Activated Protein Kinase MPK11 by the Flagellin-Derived Elicitor Peptide, flg22
}

\author{
Gerit Bethke, ${ }^{1,2}$ Pascal Pecher, ${ }^{1}$ Lennart Eschen-Lippold, ${ }^{1}$ Kenichi Tsuda, ${ }^{2}$ Fumiaki Katagiri, ${ }^{2}$ \\ Jane Glazebrook, ${ }^{2}$ Dierk Scheel, ${ }^{1}$ and Justin Lee ${ }^{1}$ \\ ${ }^{1}$ Leibniz Institute of Plant Biochemistry, Stress and Developmental Biology, Weinberg 3, D-06120 Halle, Germany; and \\ ${ }^{2}$ Department of Plant Biology, Microbial and Plant Genomics Institute, University of Minnesota, 1500 Gortner Avenue, \\ St. Paul 55108, U.S.A.
}

Submitted 8 November 2011. Accepted 16 December 2011.

\begin{abstract}
Mitogen-activated protein kinases (MAPK) mediate cellular signal transduction during stress responses, as well as diverse growth and developmental processes in eukaryotes. Pathogen infection or treatments with conserved pathogenassociated molecular patterns (PAMPs) such as the bacterial flagellin-derived flg22 peptide are known to activate three Arabidopsis thaliana MAPK: MPK3, MPK4, and MPK6. Several stresses, including flg22 treatment, are known to increase MPK11 expression but activation of MPK11 has not been shown. Here, we show that MPK11 activity can, indeed, be increased through flg22 elicitation. A small-scale microarray for profiling defense-related genes revealed that cinnamyl alcohol dehyrogenase 5 requires MPK11 for full flg22-induced expression. An mpk11 mutant showed increased flg22-mediated growth inhibition but no altered susceptibility to Pseudomonas syringae, Botrytis cinerea, or Alternaria brassicicola. In mpk3, mpk6, or mpk4 backgrounds, MPK11 is required for embryo or seed development or general viability. Although this developmental deficiency in double mutants and the lack of or only subtle mpk11 phenotypes suggest functional MAPK redundancies, comparison with the paralogous MPK4 reveals distinct functions. Taken together, future investigations of MAPK roles in stress signaling should include MPK11 as a fourth PAMP-activated MAPK.
\end{abstract}

Plants sense a changing environment and adapt accordingly by mounting responses to accommodate or counteract adverse biotic or abiotic stress conditions. At the cellular level, this requires the perception of the stress signal directly or indirectly, which is transduced through a signaling network into the final appropriate responses that typically comprise a reprogramming of biochemical activities, gene expression, or alterations of chemical content. The stress signal transduction network includes alterations in levels of various second messengers and signaling molecules such as calcium, phospholipids, reactive oxygen species, nitric oxide, or phytohormones.

The raw data for the miniarray is available in the Gene Expression Omnibus database under accession number GSE33648.

Corresponding author: J. Lee; Telephone: +49 3455582 1410; E-mail: jlee@ipb-halle.de

* The $\boldsymbol{e}$-Xtra logo stands for "electronic extra" and indicates two supplementary figures and two supplementary tables are published online.
Decoding of second messengers frequently involves phosphorylation events. For instance, calcium-dependent protein kinases (CDPK) act as a direct "sensor-transducer", where calcium binding to EF-hand motifs within the CDPK leads to activation of the kinase through conformational changes of the protein. Alternatively, calcium signals may be transduced in a two-step process, being first sensed by calcineurin B-like protein (CBL) and transmitted through altered activities of a CBL-interacting protein kinase (Batistic et al. 2009; Dodd et al. 2010). Oxidative stress signals are mediated by the oxidative signal-inducible1 (OXI1) kinase, which itself may be regulated by phospholipid signals. OXI1 has also been shown to be required for full activation of the mitogen-activated protein kinases (MAPK) MPK3 and MPK6 (Rentel et al. 2004). MAPK cascades are one of the key players in phosphorylation signaling pathways in eukaryotes (Colcombet and Hirt 2008). The prototypical MAPK cascade is a hierarchically arranged series of at least three kinases, where MAPK kinase kinase phosphorylates MAPK kinase (MKK) that then phosphorylates and activates the downstream MAPK.

In addition to stress signal transduction, MAPK cascades control several aspects of plant growth and development (Colcombet and Hirt 2008). Often, the same MAPK protein functions in diverse pathways and, thus, the signal specificities of the MAPK cascade must be tightly regulated to avoid erroneous signal crosstalk. For instance, elicitation with conserved pathogen-associated molecular patterns (PAMPs), such as the flagellin-derived flg22 elicitor peptide, activates three MAPK: MPK3, MPK6, and MPK4 (Asai et al. 2002; Bethke et al. 2009). For the immunity response, MPK3 and MPK6 are usually associated with positive regulation of the pathogen response (Asai et al. 2002). MPK4 is thought to be a negative regulator of the salicylic acid (SA)-dependent defense pathway because $m p k 4$ mutants have elevated SA levels and constitutive expression of subsets of pathogenesis-related $(P R)$ genes (Petersen et al. 2000). At the same time, these three MAPK have been associated with control of a variety of physiological processes, including stomata patterning (Wang et al. 2007), abscission process (Cho et al. 2008), ovule development (Wang et al. 2008), pollen or anther development (Hord et al. 2008), or cytokinesis (Kosetsu et al. 2010; Zeng et al. 2011). It is believed that signal specificity is, in part, controlled by scaffold proteins that maintain pathway-specific protein complexes or through distinct sets of MAPK substrates in animal and fungal systems (Kolch 2005). Presumably, the scaffold proteins or substrates are regulated by distinct spatiotemporal expression patterns that are specific for 
each pathway. In plants, no MAPK scaffolds have been reported as yet but MAPK substrates identified to date include enzymes such as 1-aminocyclopropane-1-carboxylic acid synthase- 2 or -6 that are rate-limiting enzymes in ethylene biosynthesis, or transcription factors such as ERF104, EIN3, VIP1, and several WRKYs (Andreasson and Ellis 2010), which can regulate expression of downstream target genes. The MAPK-mediated modification may affect either the activity (Cheong et al. 2003) or the protein stability of the phospho-targets (Bethke et al. 2009; Liu and Zhang 2004; Yoo et al. 2008), or it may alter protein-protein interaction dynamics (Bethke et al. 2009; Qiu et al. 2008). For example, following phosphorylation, the MPK4 substrate MKS1 is released from a preexisting complex with the WRKY33 transcription factor and MPK4, thus enabling WRKY33 to target the promoter of the PAD3 (phytoalexin-deficient 3) gene (Qiu et al. 2008). In contrast, a recent work suggests that MPK4 plays a reduced role in controlling phytoalexin accumulation during Botrytis cinerea infection, compared with MPK3 and MPK6 that can directly phosphorylate WRKY33 (Mao et al. 2011).
In addition to post-translational modification-mediated regulation of MAPK activities, MPK3 transcripts and protein levels are also increased upon stress (Ahlfors et al. 2004). It has been suggested that certain MAPK may serve as a basis for the "priming" response, where a prechallenge leads to accumulation of inactive MAPK, which can be more rapidly or more strongly activated by a second stress signal (Beckers et al. 2009). In Arabidopsis thaliana, an MPK4 paralog, MPK11, has overlapping function with MPK4 in regulating cell division but, unlike mpk4, mpk11 mutant plants are not dwarfed and do not have any obvious growth deficiencies (Kosetsu et al. 2010). Expression of MPK11 is further induced by several stress signals, including the bacterial PAMP peptide, flg22 (Genevestigator analysis). This raised the intriguing possibility that MPK11 may be stress-activated and, thus, be involved in stress responses. However, thus far, MPK11 activity has not been shown, due to either insufficient quality of the antibodies or the possibly inherent low activity of this kinase (Lee et al. 2008). This work aims to determine whether MPK11 is, indeed, a fourth MAPK activated by flg22 and, if so, how its
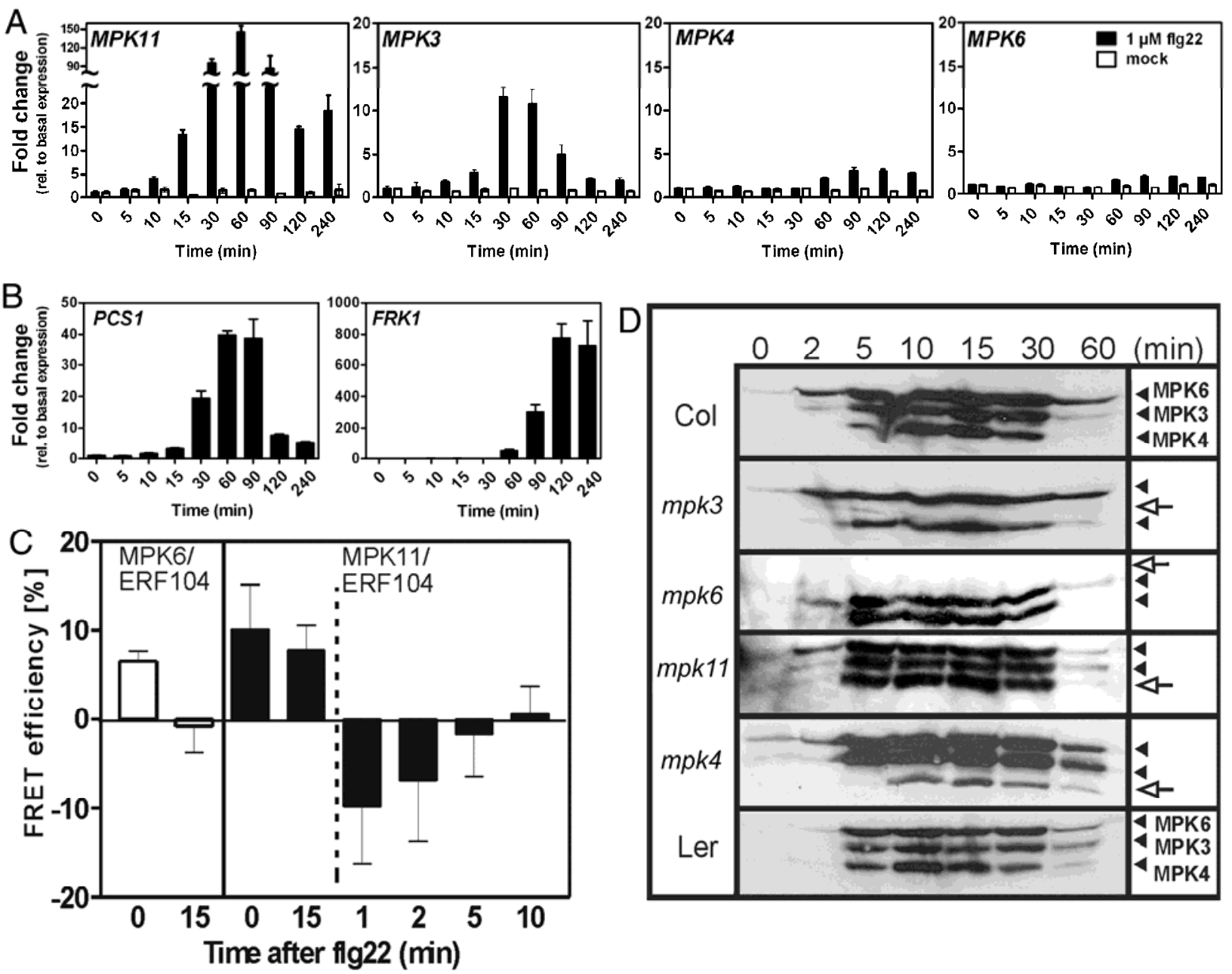

Fig. 1. Analysis of mitogen-activated protein kinase (MAPK) expression, activation, and protein-protein interaction. A, Real-time quantitative reverse-transcription polymerase chain reaction (qRT-PCR) analysis of the transcript levels of four MAPK genes after flg22 or mock (using Murashige and Skoog media) treatments is depicted as fold changes with respect to the basal expression level at time point zero. B, For comparison with the induction profile of the MAPK genes shown in A, expression of an early (phytochelatin synthase 1 [PCS1]) and late (flagellin-induced receptor kinase 1 [FRK1]) flg22-responsive gene was analyzed by qRT-PCR. C, Fluorescence resonance energy transfer (FRET) assay for detecting in vivo protein-protein interactions between an ethylene response transcription factor, ERF104, and MPK6 or MPK11, respectively, in transiently transfected protoplasts. Note that the negative FRET values are due to acceptor quenching typical for the acceptor-photobleaching-based FRET technique used here and are indicative of no physical interaction between the tested components. All error bars shown here are standard errors. D, Profiling of MAPK activation by an immunological assay that detects phosphorylation of the MAPKs. On the basis of the MAPK sizes, the expected identities of the respective immunoreactive bands are marked on the right. Open arrows mark the position of the band that should be missing in each mutant. Columbia- $0(\mathrm{Col})$ is the wild-type genotype comparison for $m p k 3, m p k 6$, and $m p k 11$, while Landsberg-erecta (Ler) is the corresponding wild-type genotype for $m p k 4$. 
function is distinct from or overlaps with MPK3, MPK6, or MPK4.

\section{RESULTS}

Flg22 effects on MPK11 expression and its function.

In silico expression analysis (Genevestigator) showed that MPK11 transcripts accumulate after diverse biotic stress conditions such as pathogen infection or treatments with PAMPs, pathogen-derived molecules, or resistance-inducing chemicals (e.g., benzothiazole), as well as several abiotic stresses such as cold, salt, hypoxia, wounding, UV, or ozone treatment (Supplementary Fig. S1). To test the PAMP-induced MPK11 expression, a time course experiment with $A$. thaliana seedlings treated with flg22 was performed. Quantitative reverse-transcription polymerase chain reaction (qRT-PCR) analysis showed that $M P K 11$ expression is, like $M P K 3$, rapidly induced within 10 to 15 min after flg22 treatment (Fig. 1A). By contrast, only a weak MPK4 and MPK6 induction (two- to threefold of the basal expression) was observed after $60 \mathrm{~min}$. For comparison, expression was tested for FRK1 (flagellin-induced receptor kinase 1) (Asai et al. 2002) and PCS1 (phytochelatin synthase 1) (Clay et al. 2009) as reference genes for late and early flg22 induction, respectively. Flg22-induced expression of MPK11 and MPK3 precedes PCS1 (Fig. 1A and B) and, hence, qualifies $M P K 11$ and $M P K 3$ as early flg22-responsive genes.
In a previous study (Bethke et al. 2009), we showed in vivo MPK6 interaction with an ethylene response transcription factor, ERF104, via a fluorescence resonance energy transfer (FRET) assay in transiently transfected protoplasts. As indicated by the loss of the FRET signals, this protein-protein interaction was disrupted 15 min after flg22 treatment of the protoplasts (Fig. 1C). MPK11 also interacted with ERF104 but the interaction was sustained despite flg22 elicitation (Fig. 1C). We routinely used 10 to $20 \mathrm{~min}$ (i.e., average $15 \mathrm{~min}$ ) of flg22 treatment because, even with minor experimental variance, maximum MAPK activation is usually attained within these time points. However, when the FRET experiment was performed with strictly defined and shorter time points, disruption of MPK11ERF104 could also be seen (Fig. 1C). Hence, MPK11-ERF104 disruption was triggered rapidly (i.e., 1 to $10 \mathrm{~min}$ ) following flg22 elicitation but, unlike MPK6-ERF104 interaction, the protein complex was "reestablished" after $15 \mathrm{~min}$ of flg22 treatment. Although the effect on MPK11 is possibly more transient, the result is a first hint that MPK11 (or its function) may be activated during flg22 treatment.

During PAMP elicitation such as flg22 treatment, increased activities for three MAPK have been detected by using peptide antibodies to specifically pull-down MPK3, MPK6, and MPK4 (Bethke et al. 2009; Meszaros et al. 2006). This activation can also be indirectly but more conveniently detected by an immunological assay based on an antibody recognizing the phosphorylated residues within the MAPK activation loop (the so-

Table 1. Selected flg22-induced genes

\begin{tabular}{|c|c|c|c|c|c|}
\hline \multirow[b]{3}{*}{ Gene loci identifier } & \multirow[b]{3}{*}{ Annotation } & \multicolumn{4}{|c|}{$\log _{2}$ differences (flg22/mock) ${ }^{a}$} \\
\hline & & \multicolumn{2}{|c|}{ Col-0 } & \multicolumn{2}{|c|}{ mpk11 } \\
\hline & & $30 \mathrm{~min}$ & $3 \mathbf{h}$ & $30 \mathrm{~min}$ & $3 \mathbf{h}$ \\
\hline \multicolumn{6}{|l|}{ Early induced genes } \\
\hline At1g80840 & WRKY4O & $4.97 *$ & $2.66^{*}$ & $5.59^{*}$ & $3.23^{*}$ \\
\hline At1g27730 & STZ (salt tolerance zinc finger) & $4.73 *$ & $2.19^{*}$ & $4.87^{*}$ & $2.42 *$ \\
\hline At4g27280 & Calcium-binding EF hand family protein & $4.64 *$ & $1.08^{*}$ & $5.21 *$ & $1.15^{*}$ \\
\hline At5g47230 & ERF5 (ethylene response factor 5) & $4.33 *$ & $1.61 *$ & $4.40^{*}$ & $1.80^{*}$ \\
\hline At2g40140 & ZFAR1 (zinc finger ankyrin repeat 1) & $4.08 *$ & $1.57^{*}$ & $4.15^{*}$ & $1.50^{*}$ \\
\hline \multicolumn{6}{|l|}{ Early (but transient) } \\
\hline At5g57560 & TCH4 (Touch 4) & $3.67 *$ & 0.26 & $4.15^{*}$ & 0.55 \\
\hline At4g31800 & WRKY18 & $2.84 *$ & 0.45 & $3.00^{*}$ & 0.65 \\
\hline At4g08950 & PHI1-like (phosphate induced1-like) & $2.40 *$ & 0.05 & $2.12 *$ & 0.02 \\
\hline At1g68840 & $R A V 2$ (AP2-domain repressor) & $2.33 *$ & 0.70 & $2.35^{*}$ & 0.89 \\
\hline At3g50060 & $M Y B 77$ & $2.32 *$ & -0.14 & $2.01 *$ & 0.11 \\
\hline \multicolumn{6}{|l|}{ Late genes } \\
\hline At2g19190 & FRK1 (flg22-induced receptor-like kinase 1) & 0.14 & $5.26^{*}$ & 0.40 & $5.63 *$ \\
\hline At4g31500 & CYP83B1 (cytochrome p450 monooxygenase) & 0.17 & $3.10^{*}$ & 0.11 & $3.63^{*}$ \\
\hline At2g37040 & PAL1 (phe ammonia lyase 1) & 0.28 & $3.01 *$ & 0.34 & $3.24 *$ \\
\hline At3g26830 & PAD3 (phytoalexin deficient 3) & -0.15 & $2.95^{*}$ & 0.02 & $3.24 *$ \\
\hline At5g54810 & TSB1 (tryptophan synthase $\beta$-subunit) & 0.75 & $2.88^{*}$ & 0.62 & $2.98 *$ \\
\hline
\end{tabular}

${ }^{a}$ Asterisks $(*)$ indicate statistically significant differences $(q<0.01)$, where $q$ is the false discovery rate-adjusted $P$ value according to the method of Storey and Tibshirani (2003).

Table 2. Genes differentially expressed in the $m p k 11$ background in microarray analysis

\begin{tabular}{|c|c|c|c|}
\hline Annotation & $\begin{array}{c}\log _{2} \text { difference, } m p k 11 / \mathrm{Col} \\
\text { (fold change) }\end{array}$ & $q$ value & qRT-PCR verification $^{\mathrm{a}}$ \\
\hline \multicolumn{4}{|l|}{ Flg22 treated (30 min) } \\
\hline At4g34230, CAD5 (cinnamyl alcohol dehydrogenase 5) & $-0.64(-1.56)$ & 0.001 & Validated \\
\hline At4g39670, Similar to ACD11 (accelerated cell death 11) & $-0.69(-1.61)$ & 0.009 & No difference \\
\hline At4g44070, PCS1 (phytochelatin synthase 1) & $0.45(1.36)$ & 0.007 & No difference \\
\hline At4g04720, PR4 (pathogenesis-related 4) & $-0.58(-1.50)$ & 0.008 & No difference \\
\hline At4g17500, AEC (auxin efflux carrier family protein) & $-0.56(-1.47)$ & 0.001 & No difference \\
\hline \multicolumn{4}{|l|}{ Mock treated } \\
\hline At4g02380, SAG21 (senescence-associated gene 21) & $-0.72(-1.65)$ & 0.007 & Not tested \\
\hline At4g18660, EXLB3 (expansin-like b3 precursor) & $-0.63(-1.55)$ & 0.004 & Not tested \\
\hline At4g09440, HSC70 (heat-shock cognate 70-kDa protein) & $-0.64(-1.56)$ & 0.003 & No difference \\
\hline
\end{tabular}


called $p$ TE $p$ Y motif, where $p$ denotes the phosphorylated residues). Three immunoreactive bands are typically detected after flg22 treatment. As seen in the corresponding mutant plants, the upper two bands correspond to MPK6 and MPK3, respectively (Fig. 1D). In an mpkl1 mutant, there is no apparent difference from the corresponding wild-type plants in the MAPK activation profile. However, in an $m p k 4$ mutant, three activated MAPK bands are still detected (albeit appearance of the lowest band is slightly delayed). Hence, we can conclude that there is at least a fourth flg22-activated MAPK of size or electrophoretic mobility similar to MPK4 in sodium dodecyl sulfate polyacrylamide gel electrophoresis.

\section{Double mpk4 mpk11 mutant is partially impaired in development.}

Because MPK4 and MPK11 are nearly identical at the amino acid sequence level (88\% identity, 92\% similarity) (Supplementary Fig. S2A) and similar in molecular weight, the third activated MAPK band detected in the immunological assay may contain both proteins. Because MPK4 and MPK11 are similar in molecular weight, the third PAMP-activated MAPK band detected in the immunological assay may contain both proteins. To test this, we crossed the mpkll mutant with the mpk4 as well as the $m p k 3$ or $m p k 6$ mutants. No mpk3 mpk11 or mpk6 mpk11 double mutants were obtained thus far. Similarly, no double mpk4 mpkll mutants were initially obtained despite screening several hundred plants. This might be due to embryo or seed lethality because empty or aborted embryos in the siliques of F2 generation plants were often found (Fig. 2A and B). However, siliques with complete rows of seeds from the mpk4 mpkll cross were occasionally detected. Thus, we screened progeny from an F4 plant that was homozygous for $m p k 11$ but heterozygous for MPK4 (i.e., $m p k 11 m p k 4^{+-}$). From 100 plants of smaller stature, as would be expected for an $m p k 4$ dwarf phenotype, three plants were unambiguously identified as $m p k 4$ mpkll double mutants. These plants were dwarfs or extreme dwarfs even as adult plants (Fig. 2C) and did not set seed (not shown). Because all four tested MAPK reside on different chromosomes, genetic linkage cannot explain the difficulty in obtaining double mutants. Taken together, our results show that, in $m p k 3, m p k 6$, or $m p k 4$ backgrounds, MPK11 is required for embryo or seed development or general viability.

\section{MPK11 is activated by flg22 elicitation.}

Leaves from the surviving mpk4 mpk11 plants were infiltrated with flg22 for $15 \mathrm{~min}$ and assayed for MAPK activation. The lowest band corresponding to the expected sizes of MPK4 or MPK11 was considerably reduced in its intensity (Fig. 2D, top panel). Hence, this confirms the speculation that the lower MAPK band contains both MPK4 and MPK11. However, a longer exposure of the blot revealed some remnant signals (Fig. 2D, bottom panel). Thus, it is possible that further MAPK of similar mobility are activated by flg 22 .

To confirm the MPK11 activation, we transiently expressed haemagglutinin (HA)-tagged MPK11 under the control of the Cauliflower mosaic virus (CaMV) $35 \mathrm{~S}$ promoter in Arabidopsis leaf protoplasts and assayed for MAPK activity following anti-HA immunoprecipitation. MPK11-HA activity increased within $5 \mathrm{~min}$ after flg22 elicitation and lasted for at least 30 min, which is distinguishable from the slight background activity in the mock immunoprecipitation experiment (Fig. 2E). Thus, MPK11 constitutes a fourth MAPK activated by flg22, in addition to MPK3, MPK4, and MPK6.

\section{Expression profiling in mpk11 plants.}

To evaluate the impact of the mpkl1 mutation on flg22responsive genes, a small-scale microarray dedicated to profil- ing Arabidopsis defense-related genes (Sato et al. 2007) was used. Two time points, $30 \mathrm{~min}$ or $3 \mathrm{~h}$ after flg22 infiltration, were chosen to cover "early" and "late" flg22-induced genes. Some examples of defense-related flg22-responsive genes are given in Table 1, showing that the elicitation was successful (a full list can be found in Supplementary Table S1). Based on a stringent significance selection of $q<0.01$ (where $q$ is the false discovery rate-adjusted $P$ value) (Storey and Tibshirani 2003), five flg22 upregulated and three downregulated genes could be detected as being differentially expressed in mpk11 compared with wild type plants (Table 2); albeit the fold differences in expression between wild type and $m p k 11$ were small. To validate the expression, we performed four independ-

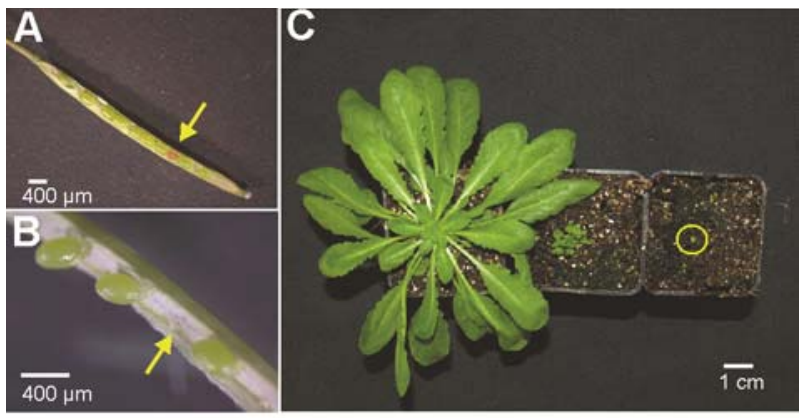

D

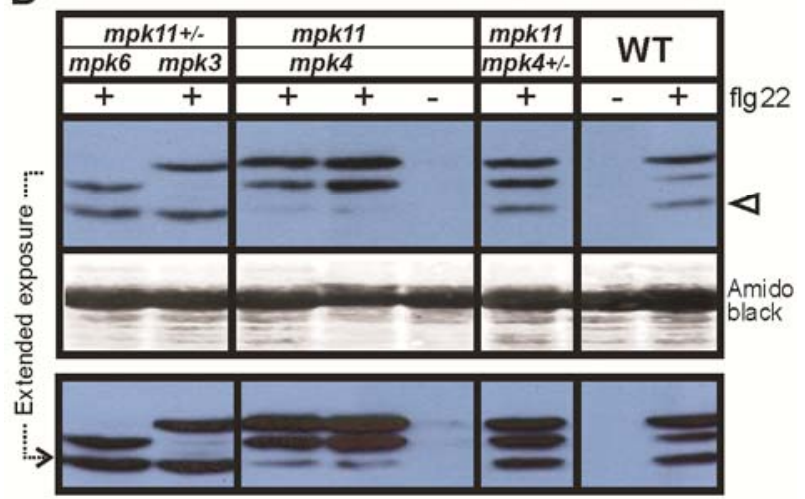

E

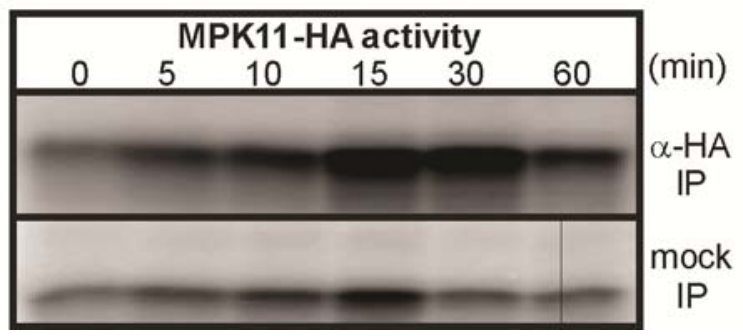

Fig. 2. Growth phenotype of progenies of $m p k 4 \mathrm{x} m p k 11$ crosses and MPK11 activity. Siliques from progeny of an $m p k 4 \times m p k 11$ cross often have $\mathbf{A}$, brownish empty seeds or $\mathbf{B}$, aborted embryos or seeds. $\mathbf{C}$, Two dwarfed $m p k 4$ mpk11 double-mutant plants are shown in comparison with a 6-week-old wild-type plant. The extreme dwarf plant is further highlighted with a yellow circle. D, Immunological assay for detecting the activated mitogen-activated protein kinases (MAPKs). Open arrowhead marks the expected position of the MPK4 or MPK11 band. Equal loading is indicated by the amido-black staining of the large subunit of Rubisco. Heterozygous genotypes are denoted by the $+/-$ symbol next to the gene allele. Note that the lower panel is an extended exposure of the same Western blot to enable the detection of weaker signals. E, MPK11 activity is revealed by immunoprecipitating haemagglutinin (HA)-tagged MPK11 in protoplasts and a subsequent radioactive assay with myelin basic protein as an artificial substrate. The mock immunoprecipitation (IP) consists of protein-G beads without the $\alpha$-HA antibodies (additional control IP experiments with $\alpha$-HA antibodies also did not immunoprecipitate any MBPphosphorylating activities from nontransfected protoplasts; not shown). The experiment was repeated at least twice with similar outcome. 
ent biological experiments and tested six of the eight genes by qRT-PCR. Despite the sensitivity of qRT-PCR, the altered expression in the mpkl1 background could not be reproducibly found for PCS1, PR4, an ACD11 (accelerated cell death 11)like gene, cinnamyl alcohol dehydrogenase 5 (CAD5), AEC

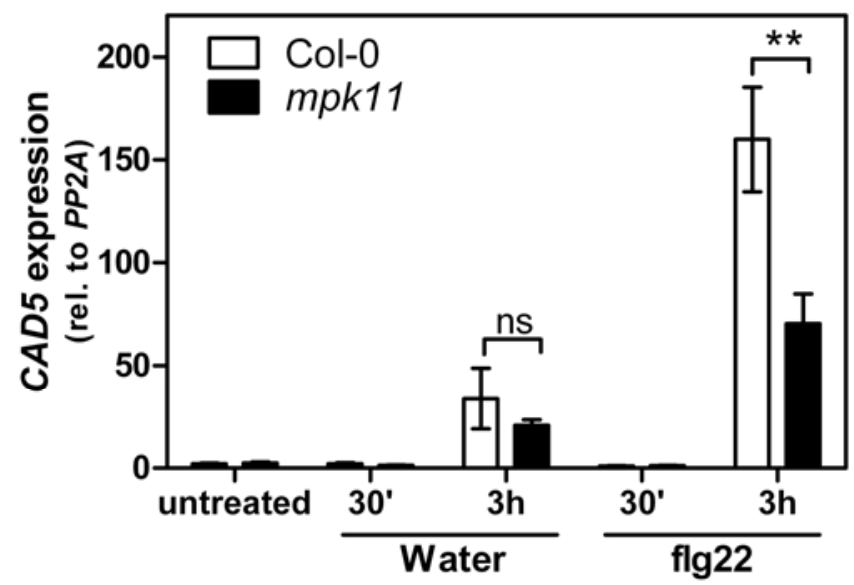

Fig. 3. Real-time quantitative polymerase chain reaction analysis of cinnamyl alcohol dehyrogenase 5 (CAD5) (At4g34230) expression. Adult 4-weekold plants were infiltrated with flg22 $(1 \mu \mathrm{M})$ or water as a mock control and harvested at the indicated time points. The relative $C A D 5$ expression was normalized to the reference gene, PP2A (At1g13320). Statistically significant differences (unpaired $t$ test with Welch's correction; ** indicates $P<0.01$ ) between expression in the Columbia-0 (Col-0) wild type and the mpk 11 mutant are indicated. A representative experiment is shown. Error bars indicate standard errors. (auxin efflux carrier) family protein, or HSC70 (heat-shock cognate 70-kDa protein) (summarized in Table 2). On the other hand, when we tested the expression of these genes in flg22infiltrated leaves of adult plants, the reduced flg22-induced expression in $m p k 11$ could be validated in independent experiments by qRT-PCR for CAD5 at $3 \mathrm{~h}$ postinfiltration (Fig. 3) but not for the other genes (data not shown). Thus, most likely, redundancies of the flg22-activated MAPK exist to compensate for lack of $M P K 11$.

\section{Contribution of MPK11}

to plant susceptibility to pathogens.

To investigate whether MPK11 affects disease resistance, infection of mpk mutant plants with phytopathogens were performed. Severity of infections by necrotrophic fungi was determined by a qPCR assay reporting the ratio of a fungal to a plant gene on the DNA level. Because the fungal DNA increases and the plant DNA decreases over the course of infection, this ratio is a quantitative estimate of disease progression. Compared with the wild-type plants, this qPCR-based assay did not detect altered susceptibility of $m p k 11, m p k 3$, or mpk6 to Alternaria brassicicola or B. cinerea (Fig. 4A and B). Similarly, no significant growth difference of the hemibiotrophic bacterial pathogen Pseudomonas syringae pv. maculicola ES4326 was observed between the Col-0 wild type compared with the $m p k$ mutants (Fig. 4C).

A prechallenge with flg22 protects plants from subsequent P. syringae pv. tomato DC3000 infection, and this PAMP-triggered immunity response requires the FLS2 receptor (Zipfel et al. 2004). As MAPK are part of the signaling cascade downstream of the FLS2 receptor, we tested whether this PAMP-
A
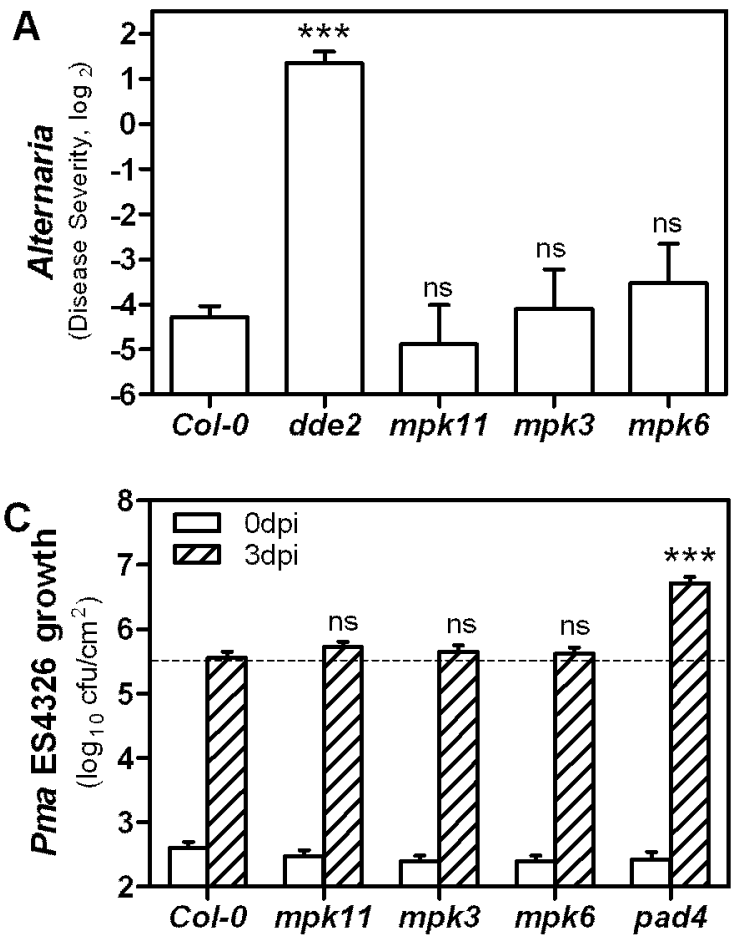
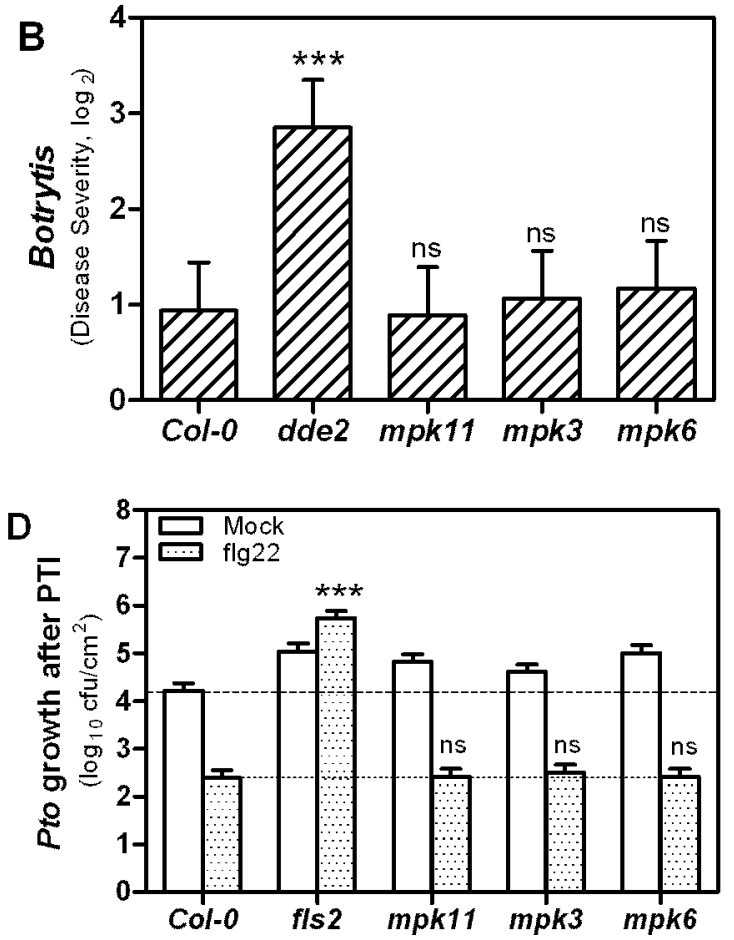

Fig. 4. Susceptibility of mitogen-activated protein kinase (MAPK) mutants to pathogens. Adult 4-week-old plants were infected with the indicated pathogens. A and $\mathbf{B}$, Progress of the necrotrophic fungi is presented as a disease severity index reporting the fungal to plant DNA $\log _{2}$ ratio. For this, DNA corresponding to the Alternaria or Botrytis CutA gene, and the plant gene AtShaggy Kinase, were determined by quantitative polymerase chain reaction. C and $\mathbf{D}$, Pseudomonas syringae growth in the leaves was determined by counting the colony-forming units (CFU) after extraction and plating on agar plates. D, Leaves were pretreated either with water (mock) or flg22 $(1 \mu \mathrm{M}) 24 \mathrm{~h}$ before challenging with $P$. syringae. Statistically significant differences (Student's $t$ test; *** indicates $P<0.001$ and ns indicates $P>0.05$ ) between the wild-type Col-0 genotype and the mutants are indicated. Delayed-dehiscence 2 (dde2), phytoalexin deficient 4 (pad4), and flagellin-sensitive 2 (fls2) mutants were used as controls for enhanced susceptibility or loss of flg22-induced pathogentriggered immunity to the pathogens, respectively. All the MAPK mutants are T-DNA insertional SALK mutants in the Col-0 ecotype. All assays have been repeated two or three times and analyzed by a mixed linear model. Error bars indicate standard errors. 
triggered immunity effect required the MAPK. However, the flg22-triggered immune protection was not affected in the tested mpk mutants (Fig. 4D). Overall, this suggests that sufficient functional redundancies likely exist between the flg22activated MAPK. Thus, any phenotypes associated with the single mpk mutants may be subtle or difficult to detect.

\section{MAPK mutants show altered growth inhibition on flg22-containing media.}

When cultivated on media containing PAMPs such as flg22, seedling growth is inhibited (Gomez-Gomez et al. 1999). In previous work on the root length of flg22-treated seedlings, we noticed a tendency of longer root length in the mpk6 mutant compared with wild-type seedlings, although the significance level was low (Bethke et al. 2009). In an effort to gain sensitivity, we repeated the assay but extended the incubation period on flg22-containing media from 14 to 20 days. This showed that $m p k 6$ roots were, indeed, longer than the wild type (Fig. $5 \mathrm{~A})$. Likewise, $m p k 4$ roots were also longer than the corresponding wild-type seedlings. By contrast, roots of both mpk3 and $m p k 11$ were significantly shorter than the Col-0 wild-type seedlings (Fig. 5A). Thus, MPK4 and MPK6 contribute to flg22-mediated root growth inhibition while MPK3 and MPK11 attenuate it.

\section{MPK4 and MPK11 have different functions despite their high sequence similarity.}

The opposite root growth arrest phenotype between $m p k 4$ and mpkl1 mutants shown above (Fig. 5A) suggests different functions of these two MAPK in flg22 signaling or in regulating defense. In contrast to reports for $m p k 4$ (Petersen et al. 2000), no enhanced expression of $P R$ genes such as $P R I$ (At2g14610), PR2 (At3g57260), or PR5 (At1g75040) was observed in the mpk11 mutant; and, similarly, basal plant defensin PDF1.2 was not repressed in mpk11. In agreement with this, levels of SA and its conjugates were not elevated in the mpk11 mutant relative to wild-type plants, whereas levels in mpk4 mutants were at least 20-fold higher (Fig. 5B). Furthermore, unlike the dwarfed mpk4, mpkll plants do not exhibit any obvious morphological differences to wild-type plants. Thus, despite the high amino acid sequence homology, MPK4 and MPK11 are not functionally analogous.

\section{DISCUSSION}

Flg22 activates more than three MAPK.

Prior to our work, three MAPK-MPK3, MPK6, and MPK4-were known to be activated upon abiotic (e.g., wound, cold, salt, or osmotic) (Droillard et al. 2004; Ichimura et al. 2000; Teige et al. 2004) and biotic (pathogen infection or PAMP treatment) (Asai et al. 2002; Bethke et al. 2009; Meszaros et al. 2006) stresses in Arabidopsis thaliana. The use of kinase assays coupled with immunoprecipitation with antibodies specific to each MAPK allowed the unequivocal demonstration of increased activities after flg22 treatment (Bethke et al. 2009; Meszaros et al. 2006). However, the high homology between MPK4 and MPK11 raises the possibility that the antibodies used might recognize both proteins. The commercially available $\alpha$-MPK4 antibody (Sigma, Schnelldorf, Germany) is targeted against the 12-amino-acid carboxyl terminal sequence of MPK4 but MPK11 is only missing the last four amino acids of this epitope. Thus, it is possible that the MPK4 activation reported thus far actually includes MPK11 activity. In accordance with this, we showed here that, in addition to increased MPK11 transcript accumulation, MPK11 is, indeed, activated upon flg22 treatment (Figs. 1 and 2). This also explains the detection of three flg22-activated MAPK bands in the $m p k 4$ or $m p k 11$ single mutants (Fig. 1D). Only in an $m p k 4$ mpk11 double mutant was the signal for this third MAPK band diminished (Fig. 2D), although there appears to be yet another weakly flg22-activated MAPK in this genetic background. Whether this implicates a fifth flg22-activable MAPK or a
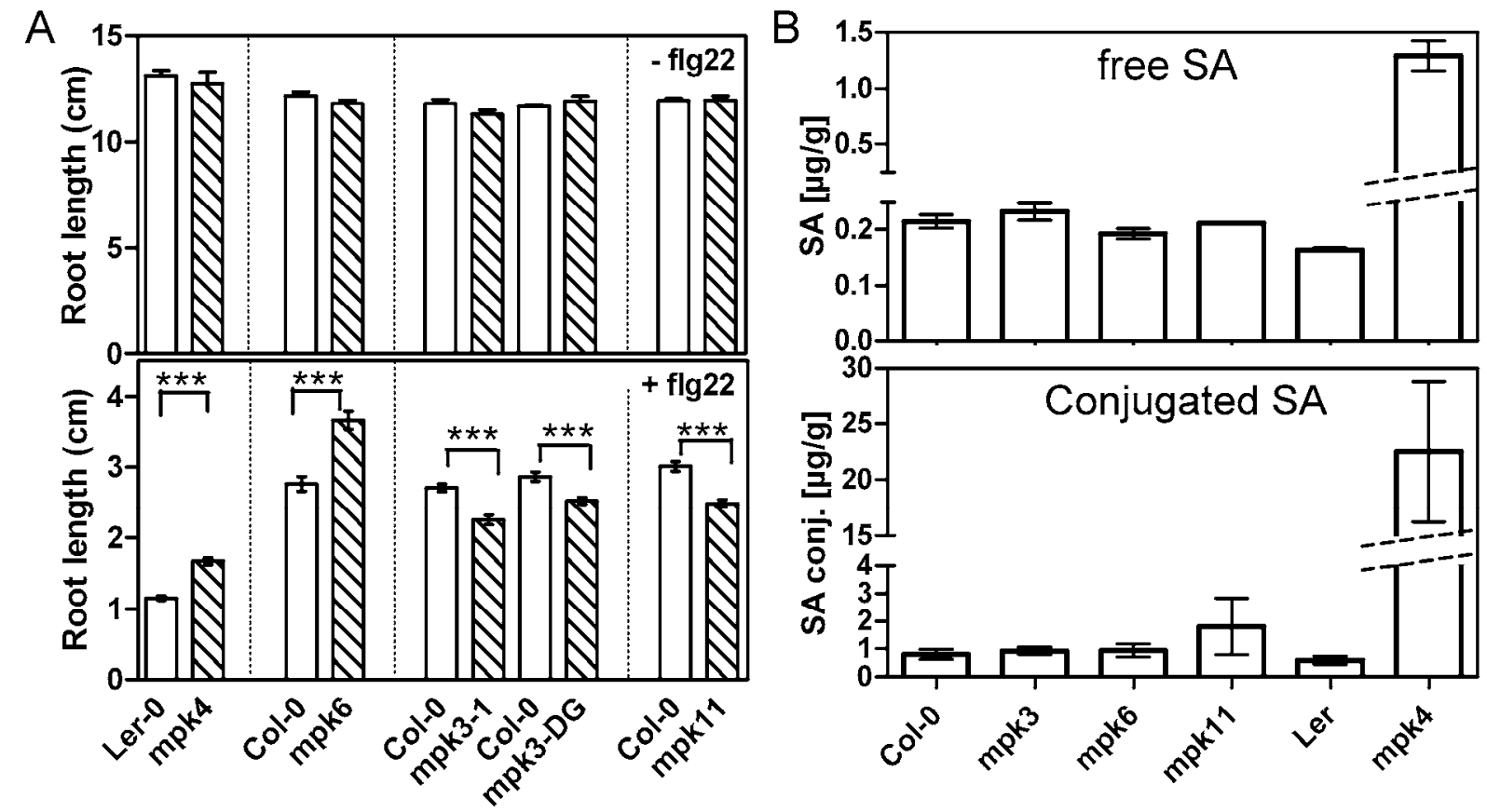

Fig. 5. Growth arrest phenotype and determination of salicylic acid (SA) levels in mitogen-activated protein kinase (MAPK) mutants. A, Root lengths were measured after growth on agar plates without flg22 (-flg22, top panel) or with (+flg22, bottom panel) for 20 days. To circumvent plate-to-plate differences, only pairwise comparisons were made (i.e., with wild-type [open bars] seedlings grown on the same plate as each mutant [hatched bars]). Statistically significant differences (Student's $t$ test; *** indicates $P<0.001$ ) are indicated. The experiment has been repeated at least three times with similar results. The $m p k 3-D G$ is a fast-neutron gene deletion $m p k 3$ mutant. B, Free SA and SA conjugates were measured in methanolic extracts of Col-0, $m p k 3$, $m p k 6$, and $m p k 11$, as well as Ler and $m p k 4$ plants grown on soil for 5 weeks. Error bars indicate standard errors. 
low-level MPK4 residual expression caused by infrequent somatic excision of the Ds transposon in the mpk4 mutant, as shown by Kosetsu and associates (2010), remains to be seen. Collectively, our work demonstrates that at least four MAPK are activated following flg22 treatment. Furthermore, expression for two of them, $M P K 3$ and $M P K 11$, is also highly induced by PAMPs such as flg22 and might have roles in induced resistance responses to future infections (Beckers et al. 2009).

\section{Distinct functions of MPK4 and MPK11.}

Despite their high sequence homology, MPK4 and MPK11 clearly have distinct functions. Unlike $m p k 4$, the $m p k 11$ mutant is neither dwarfed nor does it have elevated SA levels (Fig. 5B) and, consequently, it has normal expression levels of the SAregulated genes $P R 1, P R 2$, and $P R 5$. We showed here that mpk4 and mpk11 mutations have opposite effects on the flg22mediated growth inhibition (Fig. 5A). It was recently demonstrated that the mpk4 phenotype is not rescued by a promoter swap experiment, with MPK11 expression driven by the MPK4 promoter (Zeng et al. 2011), providing clear evidence for the functional distinction between these two paralogous genes.

\section{Role of MPK11 in cellular signaling.}

Although Zeng and associates (2011) did not obtain mpk4 mpk11 double mutants, we showed that these plants can be recovered at a low frequency. Similarly, another group showed that mpk4 mpk11 double mutants are viable but they exhibited more severe dwarfism than the mpk4 mutant (Kosetsu et al. 2010). Consistent with our results, no progeny were recovered from the double mutants. Overall, this implicates some common functions between these two kinases. Indeed, Kosetsu and associates (2010) reported that the defects in cell plate formation and growth in the mpk4 mutant were enhanced by introduction of the mpk11 mutation. Hence, despite the distinct functions discussed above, there are partially overlapping cell division functions between MPK4 and MPK11. Because viable mpk4 mpk11 plants were obtained but did not set seed, the defects may be more severe in meiotic (Zeng et al. 2011) than mitotic cytokinesis (Kosetsu et al. 2010).

Attempts to obtain mpk3 mpk6 double mutants have likewise proven challenging. Although $m p k 6^{+-} m p k 3^{--}$plants are fertile, no viable $m p k 6 m p k 3$ progeny are recovered. Plants with the reciprocal genotype $\left(m p k 6^{--} m p k 3^{+-}\right)$are female sterile (Wang et al. 2008), often fail to develop proper anthers, and produce only a small amount of viable pollen (Hord et al. 2008). Hence, $M P K 3$ is haplo-insufficient in an mpk6 background. Overall, the arrested integument development of the $m p k 6^{-1-} \mathrm{mpk}^{+/-}$ovules points to specific roles of MPK3 and MPK6 in integument cell division. Likewise, our failure to obtain $m p k 3 m p k 11$ or $m p k 6$ mpk11 double mutants indicates some functional overlap of MPK11 with MPK3 and MPK6, respectively. We currently do not know which developmental step is affected in these double mutants but, taken together, all four MAPK appear to have roles in cytokinesis during specific developmental stages (Kosetsu et al. 2010; Wang et al. 2008).

To uncover MPK11 roles in flg22 signaling, we performed a microarray designed to profile a small set of defense-regulated genes (Sato et al. 2007). We found that MPK11 is required for full flg22-induced CAD5 expression (Fig. 3). Although this initial microarray suggests some functional redundancies with other MAPK, it only covers a fraction of the genome and, therefore, more MPK11-regulated genes may be discovered upon genome-wide analysis or after other treatments (e.g., the various stress treatments known to induce accumulation of MPK11 mRNA).

CAD converts coniferyl and sinapyl aldehydes into their corresponding alcohols. CAD4 and CAD5 (also known as CAD-C and CAD-D, respectively) are the major contributors to lignin biosynthesis in Arabidopsis (Sibout et al. 2005). Interestingly, Thevenin and associates (2011) recently showed that simultaneous repression of $C A D 4 / C A D 5$ and cinnamoyl coA reductase $(C C R)$ led to sterility and dwarfism-a phenotype also observed in mpk4 mpkll plants. This is in accord with the reduced flg22-mediated CAD5 expression in mpk11. Hence, in addition to putative roles in cytokinesis, MPK11 may also affect development through altered cell wall composition.

\section{MPK11 roles in pathogen response and pathogen-triggered immunity (PTI).}

With the exception of $m p k 4$, which-possibly via the elevated SA levels - shows enhanced disease resistance (Petersen et al. 2000), reports on resistance status of the other single $m p k$ mutants are often not in complete agreement. The mpk3 mutants have larger lesions when infected with $B$. cinerea (Galletti et al. 2011; Ren et al. 2008). By contrast, MPK6 is not required for this basal immunity but for elicitor-induced resistance to $B$. cinerea (Galletti et al. 2011). In our hands, neither $m p k 3$ nor mpk6 showed enhanced susceptibility to B. cinerea or Alternaria brassicicola (Fig. 4). Although it is possible that the different methods used to determine fungal progression (Botrytis spp.-induced symptom development versus qPCR quantification) may account for this disparity, more likely reasons are environmental variations between laboratories or the use of different pathogen strains. We used B. cinerea B05.10 compared with strain DSM 4709 (Ren et al. 2008) and strain SF1 (Galletti et al. 2011). In agreement with this, phenotypical variability in growth rate was observed for different Botrytis isolates (Martinez et al. 2008).

Plants silenced for MPK6 were reported to be moderately more susceptible to virulent $P$. syringae pv. tomato and three other avirulent strains (Menke et al. 2004). By contrast, we could not show significantly altered response to $P$. syringae bacteria in the single $m p k$ mutants (Fig. 4). This variability in results between laboratories could support the notion that functional redundancies between the MAPK might mask the subtle phenotype of the single mpk mutants. In the flg22-induced root growth arrest assay, we demonstrated that statistically significant differences between mpk6 and wild-type plants can be detected by extending the incubation time with flg22 (Bethke et al. 2009) (Fig. 5). Hence, minor variations in experimental conditions may explain the lack of consensus among laboratories concerning phenotypes of single $m p k 3$ or $m p k 6$ mutants.

In spite of extensive functional redundancies among the MAPK, we could detect reduced flg22-mediated growth inhibition and CAD5 expression in the mpk11 mutant (Figs. 3 and 5). Interestingly, a cad5 mutant has been shown to be slightly more susceptible to $P$. syringae pv. tomato DC3000, and this susceptibility phenotype is further increased in a cad4 cad5 double mutant (Tronchet et al. 2010). Because the reduced $C A D 5$ expression in the mpk11 mutant is partial, finding the redundant component or components required for further regulation of $C A D 5$ (or related genes) is an intriguing possibility to manipulate resistance to $P$. syringae.

\section{Potential components of the MPK11 signaling cascade.}

Elucidating the targets of MPK11 could reveal its mode of action. Transcription factors regulating expression of CAD5 and coregulated genes could be one possibility but, currently, too few MPK11-regulated genes have been uncovered to explore this opportunity. The transient disruption of the MPK11ERF104 protein complex after flg22 elicitation (Fig. 1D) suggests that ERF104 might be a phospho-target but we have not been able to demonstrate this using in vitro kinase assays with ERF104 as a substrate (not shown). This supports previous 
conclusions that the flg22-induced disruption of the MPK6ERF104 interaction is not solely reflecting an enzyme-substrate relationship (Bethke et al. 2009). Conversely, we cannot exclude the possibility that the failure to detect ERF104 phosphorylation through MPK11 is due to the generally weak MPK11 kinase activity. MPK11 interacted with three upstream kinases (MKK1, MKK2, and MKK6) in yeast two-hybrid analysis but only weak MPK11 phosphorylation by recombinant MKK1 and MKK2 and no phosphorylation by MKK6 could be shown (Lee et al. 2008). Because phosphomodification of the active loop by its upstream kinases is essential for full MAPK activity, low MPK11 phosphorylation corresponds with a weak MPK11 activity (Kosetsu et al. 2010). Overall, the findings here and those reported elsewhere indicate that MPK11 kinase activity is comparatively weak or unstable, and may explain why it has been overlooked thus far.

In conclusion, because general growth and development are compromised in the $m p k 4 m p k 11$ genotype and, additionally, mpk3 mpk11 or mpk6 mpk11 plants appear nonviable, MPK11 shares functions with MPK4, MPK3, and MPK6 in certain signaling steps. Despite this partial redundancy, there are functional distinctions and, particularly, some role of MPK11 is evident from the altered response to flg22-induced growth inhibition of the mpk11 mutant. More importantly, MPK11 is a fourth flg22-activated MAPK and, therefore, should be included in future investigation of MAPK roles in stress responses.

\section{NOTE ADDED IN PROOF}

In agreement with our work on MPK11 activation, a recent proteomics approach showed that seven Arabidopsis MAPK, including MPK4 and possibly MPK11, are tyrosine-phosphorylated after flg22 treatment (Mithoe et al. 2011).

\section{MATERIALS AND METHODS}

\section{Plant material and growth conditions.}

Arabidopsis thaliana seeds were stratified at $4^{\circ} \mathrm{C}$ for 2 days. Plants were grown on soil in climate chambers under short day conditions $\left(22^{\circ} \mathrm{C} ; 8 \mathrm{~h}\right.$ of light, $16 \mathrm{~h}$ of darkness; $\left.140 \mu \mathrm{E}\right)$. For seedling experiments, surface-sterilized seeds were placed on half-strength Murashige and Skoog (MS) agar plates and grown under controlled conditions $\left(20\right.$ to $22^{\circ} \mathrm{C} ; 16$ of h light and $8 \mathrm{~h}$ of darkness) for 8 days. Seedlings were then transferred to liquid MS media ( $0.5 \times$ MS, $0.25 \%$ sucrose, and $1 \mathrm{mM}$ MES, $\mathrm{pH}$ 5.7) in 24-well plates (eight seedlings per well) and equilibrated for $48 \mathrm{~h}$ to allow recovery from stress caused during the transfer. The mpk6-3 (SALK127507), mpk3-1 (SALK151594), $m p k 3-D G$ (Wang et al. 2008), or mpk11 (SALK049352) (Kosetsu et al. 2010) are in the Columbia-0 ecotype, and obtained from the SALK collection via the Nottingham Arabidopsis Stock Center or from T. Spielau (University of Bayreuth, Germany) (mpk11) or B. Ellis (University of British Columbia, Canada) (mpk3-DG). The mpk4 mutant, provided by J. Mundy (University of Copenhagen, Denmark), is in the Landsbergerecta background (Petersen et al. 2000).

\section{Quantitative real-time RT-PCR.}

Seedlings in liquid MS media were elicited with $1 \mu \mathrm{M}$ flg22. As a mock control, an equivalent volume of MS medium was used. Samples were frozen at the indicated time points posttreatment. Total RNA was isolated using TRIZOL reagent according to standard protocols. DNase I-treated RNA was used for first-strand cDNA synthesis according to the manufacturer's manuals (DNase I, RNase-free; RevertAid Reverse Transcriptase; Fermentas, St. Leon-Rot, Germany). For quantitative real-time PCR, the Maxima Probe qPCR Master Mix
(Fermentas) was used. Samples were run on an Mx3005P qPCR System (Agilent Technologies, Waldbronn, Germany) Primers and real-time probes used are shown in Supplementary Table S2. The $P P 2 A$ (At1g13320) gene was used as the reference constitutively expressed gene (Czechowski et al. 2005).

\section{Microarray hybridization.}

Ten-day-old seedlings were grown in MS medium under a regime of $12 \mathrm{~h}$ of darkness and $12 \mathrm{~h}$ of light at $22^{\circ} \mathrm{C}$ and transferred to multiwell plates containing fresh liquid MS media 48 h before flg22 $(1 \mu \mathrm{M})$ or mock (water) treatment. The samples were harvested at $30 \mathrm{~min}$ and $3 \mathrm{~h}$. Three independent replicates for each time point/treatment were used. Microarray hybridization and data analysis were performed as previously described (Sato et al. 2007, 2010). Briefly, the normalized hybridization signal values were subjected to the following linear model analysis via the lmFit function in the limma package in the $\mathrm{R}$ environment: $\log 2$ (expression level value) $\approx$ genotype/ treatment/time + replicate.

The eBayes function in the limma package was used for variance shrinkage in the calculation of the $P$ values and the Storey's $q$ values were calculated using the $q$ value function (Storey and Tibshirani 2003).

\section{Protein work.}

For leaf infiltration experiments, 4- to 5-week-old plants were infiltrated with either $1 \mu \mathrm{M}$ flg22 or water as control. Protein extraction, immunoblot analysis ( $\alpha$-phospho-p44/42ERK; Cell Signaling Technology, distributed by New England Biolabs, Frankfurt, Germany), and immunoprecipitation experiments ( $\alpha$-HA.11; Eurogentec, Cologne, Germany) were performed as described (Ahlfors et al. 2004).

\section{Protoplast isolation, transfection, and FRET analysis.}

Protoplast isolation, transfection, immunoprecipitation, and FRET experiments were performed as previously described (Bethke et al. 2009; Ranf et al. 2011), with all tested components under the control of the CaMV $35 \mathrm{~S}$ promoter.

\section{Pathogen assays.}

Infection experiments with the fungal pathogens $B$. cinerea B05.10 and Alternaria brassicicola ATTC 96836 were performed as described by Gachon and Saindrenan (2004), Flors and associates (2008), and Bethke and associates (2009), with slight modifications. A. brassicicola was grown for 10 days on plates containing a modified potato dextrose agar (PDA) medium (PDA at $19 \mathrm{~g} /$ liter, sucrose at $20 \mathrm{~g} / \mathrm{liter}, \mathrm{CaCO}_{3}$ at 30 $\mathrm{g} /$ liter, and agar at $7.5 \mathrm{~g} / \mathrm{liter}$ ). Spores were harvested, filtered through cheese cloth, and adjusted to a concentration of $5 \times$ $10^{5}$ spores $/ \mathrm{ml}$ in Alternaria inoculation medium $(1 \times$ Gamborg B5 [Sigma], $10 \mathrm{mM}$ sucrose, and $10 \mathrm{mM} \mathrm{KH}_{2} \mathrm{PO}_{4}$ ). B. cinerea was cultivated for 2 weeks on $1 \times$ PDA plates and spores were harvested, filtered through cheese cloth, and adjusted to $2 \times$ $10^{5}$ spores $/ \mathrm{ml}$ in Botrytis inoculation medium (1× Gamborg B5 [Sigma], $2 \%$ glucose, and $10 \mathrm{mM} \mathrm{K}_{3} \mathrm{PO}_{4}$ [pH 6.4]). Alternaria and Botrytis spp. infections were done by placing $10-\mu l$ drops of spore solutions onto leaves of 3- or 4-week-old plants, respectively. Leaf disks of the infected region were harvested 7 dpi for A. brassicicola and $3 \mathrm{dpi}$ for $B$. cinerea. The pathogenspecific primers used in quantification of the fungal growth were adopted from Gachon and Saindrenan (2004).

For the infiltration of $P$. syringae pv. tomato DC3000 or $P$. syringae pv. maculicola ES4326, the experimental setup consisted of eight pots per tray, with typically up to eight different genotypes in one pot. Two leaves of the 30- to 31-day-old plants were infiltrated with bacteria resuspended at and optical density at $600 \mathrm{~nm}=0.0001$. Two leaf discs per leaf (total leaf 
area $=0.57 \mathrm{~cm}^{2}$ ) were pooled to obtain one sample per leaf. Two of the pots per tray were used for day 0 and the remaining six pots were used for the other time points. This resulted in 4 replicates per experiment for $0 \mathrm{dpi}$ and 12 replicates per experiment for 3 dpi.

For the PTI assay, plants were pretreated with $1 \mu \mathrm{M}$ flg22 (or water as a mock control) and infiltrated $24 \mathrm{~h}$ later with $P$. syringae pv. tomato DC3000, as described (Zipfel et al. 2004). The flagellin-sensitive 2 ( fls2) mutant was used as a control for the PTI experiments.

For all pathogen experiments, the data were collected from two or more independent biological replicates and combined for analysis using a mixed linear model as described (Tsuda et al. 2008).

\section{SA measurements.}

SA and SA conjugates were extracted according to Verberne and associates (2002). Samples were measured with an Acquity UPLC (Waters Corp., Eschborn, Germany) (Eschen-Lippold et al. 2010).

\section{Root growth inhibition assays.}

Root growth assays were performed as described (Bethke et al. 2009; Ranf et al. 2011).

\section{Statistical analyses.}

$\mathrm{R}$ and GraphPad Prism 5 software were used for statistical analyses.

\section{ACKNOWLEDGMENTS}

We thank N. Bauer and C. Rülke for excellent technical support. This work is supported by the Deutsche Forschungsgemeinschaft program, SFB648, and the Bundesministerium für Bildung und Forschung program, ProNET-T3, to D. Scheel and J. Lee; a grant (DE-FG02-09ER15670) to J. Glazebrook from the Chemical Sciences, Geosciences and Biosciences Division, Office of Basic Energy Sciences, Office of Science, U.S. Department of Energy; and National Science Foundation grant MCB-0918908 to F. Katagiri.

\section{LITERATURE CITED}

Ahlfors, R., Macioszek, V., Rudd, J., Brosche, M., Schlichting, R., Scheel, D., and Kangasjarvi, J. 2004. Stress hormone-independent activation and nuclear translocation of mitogen-activated protein kinases in Arabidopsis thaliana during ozone exposure. Plant J. 40:512-522.

Andreasson, E., and Ellis, B. 2010. Convergence and specificity in the Arabidopsis MAPK nexus. Trends Plant Sci. 15:106-113.

Asai, T., Tena, G., Plotnikova, J., Willmann, M. R., Chiu, W. L., GomezGomez, L., Boller, T., Ausubel, F. M., and Sheen, J. 2002. MAP kinase signalling cascade in Arabidopsis innate immunity. Nature 415:977983.

Batistic, O., Waadt, R., Steinhorst, L., Held, K., and Kudla, J. 2009. CBLmediated targeting of CIPKs facilitates the decoding of calcium signals emanating from distinct cellular stores. Plant J. 61:211-222.

Beckers, G. J., Jaskiewicz, M., Liu, Y., Underwood, W. R., He, S. Y., Zhang, S., and Conrath, U. 2009. Mitogen-activated protein kinases 3 and 6 are required for full priming of stress responses in Arabidopsis thaliana. Plant Cell 21:944-953.

Bethke, G., Unthan, T., Uhrig, J. F., Poschl, Y., Gust, A. A., Scheel, D., and Lee, J. 2009. Flg22 regulates the release of an ethylene response factor substrate from MAP kinase 6 in Arabidopsis thaliana via ethylene signaling. Proc. Natl. Acad. Sci. U.S.A. 106:8067-8072.

Cheong, Y. H., Moon, B. C., Kim, J. K., Kim, C. Y., Kim, M. C., Kim, I.H., Park, C. Y., Kim, J. C., Park, B. O., Koo, S. C., Yoon, H. W., Chung, W. S., Lim, C. O., Lee, S. Y., and Cho, M. J. 2003. BWMK1, a rice mitogen-activated protein kinase, locates in the nucleus and mediates pathogenesis-related gene expression by activation of a transcription factor. Plant Physiol. 132:1961-1972.

Cho, S. K., Larue, C. T., Chevalier, D., Wang, H., Jinn, T. L., Zhang, S., and Walker, J. C. 2008. Regulation of floral organ abscission in Arabidopsis thaliana. Proc. Natl. Acad. Sci. U.S.A. 105:15629-15634.

Clay, N. K., Adio, A. M., Denoux, C., Jander, G., and Ausubel, F. M. 2009.
Glucosinolate metabolites required for an Arabidopsis innate immune response. Science 323:95-101.

Colcombet, J., and Hirt, H. 2008. Arabidopsis MAPKs: A complex signalling network involved in multiple biological processes. Biochem. J. 413:217-226.

Czechowski, T., Stitt, M., Altmann, T., Udvardi, M. K., and Scheible, W. R. 2005. Genome-wide identification and testing of superior reference genes for transcript normalization in Arabidopsis. Plant Physiol. 139:5-17.

Dodd, A. N., Kudla, J., and Sanders, D. 2010. The Language of calcium signalling. Annu. Rev. Plant Biol. 61:593-620.

Droillard, M. J., Boudsocq, M., Barbier-Brygoo, H., and Lauriere, C. 2004. Involvement of MPK4 in osmotic stress response pathways in cell suspensions and plantlets of Arabidopsis thaliana: Activation by hypoosmolarity and negative role in hyperosmolarity tolerance. FEBS (Fed. Eur. Biochem. Soc.) Lett. 574:42-48.

Eschen-Lippold, L., Altmann, S., and Rosahl, S. 2010. DL-beta-aminobutyric acid-induced resistance of potato against Phytophthora infestans requires salicylic acid but not oxylipins. Mol. Plant-Microbe Interact. 23:585-592.

Flors, V., Ton, J., van Doorn, R., Jakab, G., Garcia-Agustin, P., and Mauch-Mani, B. 2008. Interplay between JA, SA and ABA signalling during basal and induced resistance against Pseudomonas syringae and Alternaria brassicicola. Plant J. 54:81-92.

Gachon, C., and Saindrenan, P. 2004. Real-time PCR monitoring of fungal development in Arabidopsis thaliana infected by Alternaria brassicicola and Botrytis cinerea. Plant Physiol. Biochem. 42:367-371.

Galletti, R., Ferrari, S., and De Lorenzo, G. 2011. Arabidopsis MPK3 and MPK6 play different roles in basal and oligogalacturonide- or flagellininduced resistance against Botrytis cinerea. Plant Physiol. 157:804-814.

Gomez-Gomez, L., Felix, G., and Boller, T. 1999. A single locus determines sensitivity to bacterial flagellin in Arabidopsis thaliana. Plant J. 18:277-284.

Hord, C. L., Sun, Y. J., Pillitteri, L. J., Torii, K. U., Wang, H., Zhang, S., and Ma, H. 2008. Regulation of Arabidopsis early anther development by the mitogen-activated protein kinases, MPK3 and MPK6, and the ERECTA and related receptor-like kinases. Mol. Plant 1:645-658.

Ichimura, K., Mizoguchi, T., Yoshida, R., Yuasa, T., and Shinozaki, K. 2000. Various abiotic stresses rapidly activate Arabidopsis MAP kinases ATMPK4 and ATMPK6. Plant J. 24:655-665.

Kolch, W. 2005. Coordinating ERK/MAPK signalling through scaffolds and inhibitors. Nat. Rev. Mol. Cell Biol. 6:827-837.

Kosetsu, K., Matsunaga, S., Nakagami, H., Colcombet, J., Sasabe, M., Soyano, T., Takahashi, Y., Hirt, H., and Machida, Y. 2010. The MAP kinase MPK4 is required for cytokinesis in Arabidopsis thaliana. Plant Cell 22:3778-3790.

Lee, J. S., Huh, K. W., Bhargava, A., and Ellis, B. E. 2008. Comprehensive analysis of protein-protein interactions between Arabidopsis MAPKs and MAPK kinases helps define potential MAPK signalling modules. Plant Signal. Behav. 3:1037-1041.

Liu, Y., and Zhang, S. 2004. Phosphorylation of 1-aminocyclopropane-1carboxylic acid synthase by MPK6, a stress-responsive mitogen-activated protein kinase, induces ethylene biosynthesis in Arabidopsis. Plant Cell 16:3386-3399.

Mao, G., Meng, X., Liu, Y., Zheng, Z., Chen, Z., and Zhang, S. 2011. Phosphorylation of a WRKY transcription factor by two pathogenresponsive MAPKs drives phytoalexin biosynthesis in Arabidopsis. Plant Cell 23:1639-1653.

Martinez, J. A., Valdes, R., Vicente, M. J., and Banon, S. 2008. Phenotypical differences among B. cinerea isolates from ornamental plants. Commun. Agric. Appl. Biol. Sci. 73:121-129.

Menke, F. L., van Pelt, J. A., Pieterse, C. M., and Klessig, D. F. 2004. Silencing of the mitogen-activated protein kinase MPK6 compromises disease resistance in Arabidopsis. Plant Cell 16:897-907.

Meszaros, T., Helfer, A., Hatzimasoura, E., Magyar, Z., Serazetdinova, L., Rios, G., Bardoczy, V., Teige, M., Koncz, C., Peck, S., and Bogre, L. 2006. The Arabidopsis MAP kinase kinase MKK1 participates in defence responses to the bacterial elicitor flagellin. Plant J. 48:485-498.

Mithoe, S. C., Boersema, P. J., Berke, L., Snel, B., Heck, A. J., and Menke, F. L. H. 2011. Targeted quantitative phosphoproteomics approach for the detection of phospho-tyrosine signaling in plants. J. Proteome Res. 11:438-448.

Petersen, M., Brodersen, P., Naested, H., Andreasson, E., Lindhart, U., Johansen, B., Nielsen, H. B., Lacy, M., Austin, M. J., Parker, J. E., Sharma, S. B., Klessig, D. F., Martienssen, R., Mattsson, O., Jensen, A. B., and Mundy, J. 2000. Arabidopsis map kinase 4 negatively regulates systemic acquired resistance. Cell 103:1111-1120.

Qiu, J. L., Fiil, B. K., Petersen, K., Nielsen, H. B., Botanga, C. J., Thorgrimsen, S., Palma, K., Suarez-Rodriguez, M. C., SandbechClausen, S., Lichota, J., Brodersen, P., Grasser, K. D., Mattsson, O., Glazebrook, J., Mundy, J., and Petersen, M. 2008. Arabidopsis MAP 
kinase 4 regulates gene expression through transcription factor release in the nucleus. EMBO (Eur. Mol. Biol. Organ.) J. 27:2214-2221.

Ranf, S., Eschen-Lippold, L., Pecher, P., Lee, J., and Scheel, D. 2011. Interplay between calcium signalling and early signalling elements during defence responses to microbe- or damage-associated molecular patterns. Plant J. 68:100-113.

Ren, D., Liu, Y., Yang, K. Y., Han, L., Mao, G., Glazebrook, J., and Zhang, S. 2008. A fungal-responsive MAPK cascade regulates phytoalexin biosynthesis in Arabidopsis. Proc. Natl. Acad. Sci. U.S.A. 105:5638-5643.

Rentel, M. C., Lecourieux, D., Ouaked, F., Usher, S. L., Petersen, L., Okamoto, H., Knight, H., Peck, S. C., Grierson, C. S., Hirt, H., and Knight, M. R. 2004. OXI1 kinase is necessary for oxidative burst-mediated signalling in Arabidopsis. Nature 427:858-861.

Sato, M., Mitra, R. M., Coller, J., Wang, D., Spivey, N. W., Dewdney, J., Denoux, C., Glazebrook, J., and Katagiri, F. 2007. A high-performance, small-scale microarray for expression profiling of many samples in Arabidopsis-pathogen studies. Plant J. 49:565-577.

Sato, M., Tsuda, K., Wang, L., Coller, J., Watanabe, Y., Glazebrook, J., and Katagiri, F. 2010. Network modeling reveals prevalent negative regulatory relationships between signaling sectors in Arabidopsis immune signaling. PLoS Pathog. 6:e1001011. Published online.

Sibout, R., Eudes, A., Mouille, G., Pollet, B., Lapierre, C., Jouanin, L., and Seguin, A. 2005. CINNAMYL ALCOHOL DEHYDROGENASE-C and $-\mathrm{D}$ are the primary genes involved in lignin biosynthesis in the floral stem of Arabidopsis. Plant Cell 17:2059-2076.

Storey, J. D., and Tibshirani, R. 2003. Statistical significance for genomewide studies. Proc. Natl. Acad. Sci. U.S.A. 100:9440-9445.

Teige, M., Scheikl, E., Eulgem, T., Doczi, R., Ichimura, K., Shinozaki, K., Dang1, J. L., and Hirt, H. 2004. The MKK2 pathway mediates cold and salt stress signaling in Arabidopsis. Mol. Cell 15:141-152.

Thevenin, J., Pollet, B., Letarnec, B., Saulnier, L., Gissot, L., MaiaGrondard, A., Lapierre, C., and Jouanin, L. 2011. The simultaneous repression of CCR and CAD, two enzymes of the lignin biosynthetic pathway, results in sterility and dwarfism in Arabidopsis thaliana. Mol. Plant 4:70-82.

Tronchet, M., Balague, C., Kroj, T., Jouanin, L., and Roby, D. 2010. Cinnamyl alcohol dehydrogenases-C and $\mathrm{D}$, key enzymes in lignin biosyn- thesis, play an essential role in disease resistance in Arabidopsis. Mol. Plant Pathol. 11:83-92.

Tsuda, K., Sato, M., Glazebrook, J., Cohen, J. D., and Katagiri, F. 2008 Interplay between MAMP-triggered and SA-mediated defense responses. Plant J. 53:763-775.

Verberne, M. C., Brouwer, N., Delbianco, F., Linthorst, H. J., Bol, J. F., and Verpoorte, R. 2002. Method for the extraction of the volatile compound salicylic acid from tobacco leaf material. Phytochem. Anal. 13:45-50.

Wang, H., Ngwenyama, N., Liu, Y., Walker, J. C., and Zhang, S. 2007. Stomatal development and patterning are regulated by environmentally responsive mitogen-activated protein kinases in Arabidopsis. Plant Cell 19:63-73.

Wang, H., Liu, Y., Bruffett, K., Lee, J., Hause, G., Walker, J. C., and Zhang, S. 2008. Haplo-insufficiency of MPK3 in MPK6 mutant background uncovers a novel function of these two MAPKs in Arabidopsis ovule development. Plant Cell 20:602-613.

Yoo, S. D., Cho, Y. H., Tena, G., Xiong, Y., and Sheen, J. 2008. Dual control of nuclear EIN3 by bifurcate MAPK cascades in C2H4 signalling. Nature 451:789-795.

Zeng, Q., Chen, J. G., and Ellis, B. E. 2011. AtMPK4 is required for malespecific meiotic cytokinesis in Arabidopsis. Plant J. 67:895-906.

Zipfel, C., Robatzek, S., Navarro, L., Oakeley, E. J., Jones, J. D., Felix, G., and Boller, T. 2004. Bacterial disease resistance in Arabidopsis through flagellin perception. Nature 428:764-767.

\section{AUTHOR-RECOMMENDED INTERNET RESOURCES}

The Arabidopsis Information Resource (TAIR) website: www.arabidopsis.org

European Arabidopsis Stock Centre (NASC) database: arabidopsis.info Genevestigator microarray database: www.genevestigator.com/gv

GraphPad software website: www.graphpad.com

R software: cran.r-project.org/

Salk Institute Genomic Analysis Laboratory SIGnAL database: signal.salk.edu

In the originally published version, the Western blot panel for the Landsberg-erecta (Ler) genotype was erroneously duplicated and depicted as the mpkl1 mutant in Figure 1D. The figure was changed on February 18, 2015, to present the correct compiled figure showing the phosphorylated forms of the MAPKs (mitogen-activated protein kinases). 